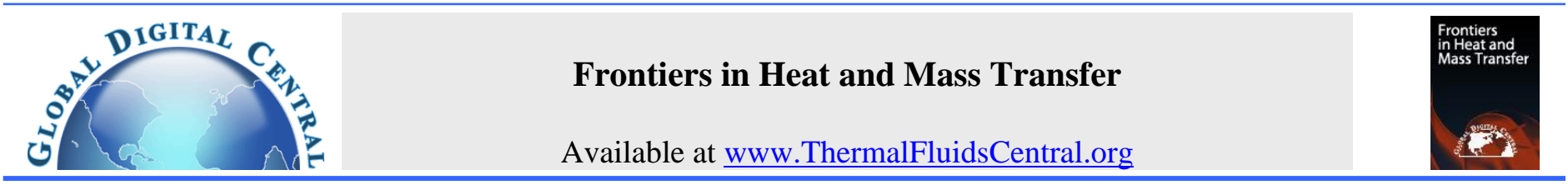

\title{
UNSTEADY MHD BLASIUS AND SAKIADIS FLOWS WITH VARIABLE THERMAL CONDUCTIVITY IN THE PRESENCE OF THERMAL RADIATION AND VISCOUS DISSIPATION
}

\author{
Stanford Shateyi ${ }^{\mathrm{a} *}$ and Hillary Muzara ${ }^{\mathrm{b}}$ \\ ${ }^{a}$ University of Venda, Department of Mathematics, P Bag X5050, Thohoyandou 0950, South Africa. \\ ${ }^{\mathrm{b}}$ University of Zimbabwe, Department of Mathematics, P O Box MP167, Harare, Zimbabwe
}

\begin{abstract}
A theoretical analysis has been carried out to investigate the influence of unsteadiness on the laminar two-phase magnetohydrodynamic nanofluid flow filled with porous medium under the combined effects of Brownian motion and thermophoresis. Thermal variable conductivity, thermal radiation and viscous dissipation effects are also considered in this numerical study. The highly nonlinear partial differential equations are transformed into a set of coupled nonlinear ordinary differential equations through suitable similarity transformations. The resultant ordinary differential equations are then numerically solved using the spectral quasilinearization method. The effects of the pertinent physical parameters over the fluid velocity, temperature, concentration, skin friction, Nusselt and Sherwood numbers for both Blasius and Sakiadis flows are presented in graphical and tabular forms and discussed. It is found that the two types of flows behave differently when the physical parameters are varied.
\end{abstract}

Keywords: Brownian motion, thermophoresis, spectral quasilinearization method.

\section{INTRODUCTION}

Several researchers have been attracted to investigate electrically conducting fluid flows due to their day-to-day applications in numerous engineering areas, such as paper production, cooling of metallic sheets, plasma studies, petroleum industries, cooling of nuclear reactors and magnetohydrodynamic power generators. Blasius (1908) was the first to solve the problem of the fluid flow along a horizontal stationary semi-infinite plate in a moving with a constant velocity. This gave birth to what is now widely known as Blasius boundary layer flow. The fluid motion in a Blasius problem is produced by the free stream. Howarth (1938) later found out a numerical solution to the Blasius problem. The desired cooling effect can be achieved by controlling the rate of heat transfer through the application of the MHD flow in electrically conducting fluid. Further, Lighthill (1950) discussed the theory of heat transfer through a laminar boundary layer. Liu and Chang Liu and Chang (2008), later developed a numerical technique by using new transformation that transforms the governing equation into a nonlinear second-order- boundary value problem. Aminikhah and Kazemi (2016) employed the elastic B-spline approximations to construct to a numerical solution for solving the Blasius equation.

In 1961, Sakiadis (1961) improved the Blasius problem to consider the flow over a moving plate or sheet through an otherwise environment of a quiescent fluid. Through his pioneering work, solutions to this different class of boundary layer problems are significantly different from those of boundary layer flows over stationary surfaces. Subsequently, Tsou et al. (1967) managed to experimentally and analytically showed that such flows are physically realizable by confirming the data from Sakiadis. Since then, many researchers have obtained analytical/numerical solutions for different aspects of this class of boundary-layer problems. This class of boundary-layer problem is now known as Sakiadis boundary layer flow. Crane (1970) studied the flow over a stretching sheet which moves in its own plane at a velocity that varies linearly with the distance from the slit. Wang (2004) solved the classical Blasius equation using the Adomian decomposition method. Chamkha (2004) investigated the problem of unsteady, two dimensional, laminar boundary layer flow of a viscous, incompressible electrically conducting and heat fluid over a semiinfinite plate. Choi (1995) proposed the suspension of nanoparticles in a base fluid like water, Ethylene glycol and glycol. This gave birth to a type of fluid flow now know as nanofluids. It is known that conventional fluids such as water, are poor heat transfer fluids. Nanofluids have the potential to reduce thermal resistances and industrial groups such as electronics, medical, food and manufacturing are benefiting from these improved heat transfer. Nanotechnology has been an ongoing hot topic health as researchers claim that nanoparticles could present possible dangers in environment, Mnyusiwalla et al. (2003). Sharma et al. (2017) investigated the steady two dimensional laminar magnetohydrodynamic slip flow and heat transfer of a viscous incompressible and electrically conducting fluid past a flat exponentially non-conducting stretching porous sheet.

\footnotetext{
*University of Venda, Department of Mathematics, P Bag X5050, Thohoyandou 0950, South Africa.

${ }^{\dagger}$ Corresponding author.Email: stanford.shateyi@univen.ac.za
} 
Buongiorno (2006) explained how the increase in thermal conductivity of nanofluids and also developed a model that considered the particle Brownian motion and thermophoresis. Kuznetsov and Nield (2010) investigated the effects of Brownian motion and thermophoresis on the natural convective boundary layer flow of a nanofluid. Oyelakin et al. (2016) reported the combing Dufour and Soret effects on the heat and mass transfer in a Casson nanofluid flow over an unsteady stretching sheet with thermal radiation and heat generation. The study also investigated the effects of partial slip, convective thermal boundary condition, Brownian and thermophoresis diffusion coefficients. Ahmad and Ishak (2017) studied a Blasius and Sakiadis problem in nanofluids. The Buongiormo model was used by Anuar and Bachok (2016) to investigate thermophysical properties of nano-liquids due to Blasius and Sakiadis problem. Hady et al. (2013) investigated the density effects on the flow and heat transfer characteristics of the Blasius and Sakiadis flow in a nanofluid in the presence of thermal radiation under convective surface boundary condition. Sekhar et al. (2017) theoretically analysed the flow of heat transfer characteristics of Sakiadus and Blasius flow of an MHD Maxwell fluid with an exponential heat source/sink. Cortell (2005) discussed the problem in Blasius and Sakiadis by considering thermal radiation effects with convective surface boundary conditions. Mabood et al. (2016) theoretically investigated the effects of volume fraction of nanoparticles, suction/injection and convective heat and mass transfer parameter on MHD stagnation point flow of water based nanofluid ( $\mathrm{Cu}$ and $\mathrm{Ag}$ ). Narsu and Kumar (2018) investigated the effects of unsteady MHD chemically reacting radiated Blasius with variable conductivity and a Sakiadis flow under a convective surface boundary condition. Reddy and B. Mallikarjuna (2018) developed a mathematical model to analyze the double diffusive convective of Casson fluid over an inclined stretching sheet with Cattaneo-Christov Heat Flux model. Recently, Mjankwi et al. (2019) studied the unsteady MHD flow of a nanofluid with variable fluid properties over an inclined stretching sheet in the presence of thermal radiation and chemical reaction taking into account variable thermal conductivity and diffusion coefficient. Krishna et al. (2019) theoretically studied the effect of MHD field on the classical Blasius and Sakiadis flows of heat transfer characteristics with variable conditions and variable properties. ? studied the effect of non-uniform slot suction or injection into a steady mixed convective MHD boundary layer flow over a vertical wedge embedded in a porous medium. Astuti et al. (2019) attempted to provide information and limitations of the use of nanofluids in designing a cooling system in the ingineering field.

Motivated by the above studies, the current study aims at investigating the unsteady laminar two phase (Blasius and Sakiadis) MHD nanofluid flow filled with porous medium under the combined effects of Brownian motion and thermophoresis as well as variable thermal conductivity. The innovation of this paper lies in the unification of more factors into the governing equations and an attempt to give a thorough analysis of how the flow properties are affected by these factors. Also the novelty of the current paper lies in the application of the recently developed numerical method to solve these highly nonlinear equations. In this research this governing equations which are highly nonlinear partial differential equations are transformed into nonlinear ordinary differential equations. The newly developed spectral quasi-linearization method will be applied to obtain numerical solutions to the current model. The effects or influences of nondimensional governing pertinent parameter on the dimensionless velocity, temperature and concentration profiles as well skin friction, Nusselt number and Sherwood number will be discussed with the aid of graphs and/or tables.

\section{MATHEMATICAL FORMULATION}

We consider the unsteady laminar two-phase (Blasius and Sakiadis) flow filled with porous media under the combined effects of Brownian motion and thermophoresis as well as thermal radiation. Variable thermal conductivity is also considered in this study. The cartesian coordinate has the origin at the leading edge with the $x$-axis extending along the sheet in the flow direction and the $y$-axis is measured perpendicular to the flow. The unsteady fluid flow, heat and mass transfer start at time $(t)=0$ and the sheet is being stretched with velocity $U_{w}(x, t)$ along the $x$-axis while the origin is kept fixed.

The temperature and the concentration fields of the sheet are respectively, $T_{w}(x, t)$ and $C_{w}(x, t)$ and are summed to be linear functions of $x$. The thermo-physical properties of the sheet and the ambient fluid are assumed to be constant except density variations and thermal conductivity which we assume to be linearly varying with temperature. Under these assumptions together with the Boussinesq and boundary layer approximations, the governing equations for the model under consideration are:

$$
\begin{gathered}
\frac{\partial u}{\partial x}+\frac{\partial v}{\partial y}=0, \\
\frac{\partial u}{\partial t}+u \frac{\partial u}{\partial x}+v \frac{\partial u}{\partial y}=\nu \frac{\partial^{2} u}{\partial y^{2}} \pm g \beta\left(T-T_{\infty}\right) \pm g \beta_{c}\left(C-C_{\infty}\right) \\
-\frac{\sigma B_{0}^{2}}{\rho} u-\frac{\varphi}{K_{o}} u \\
\frac{\partial T}{\partial t}+u \frac{\partial T}{\partial x}+v \frac{\partial T}{\partial y}=\frac{1}{\rho c_{p}} \frac{\partial}{\partial y}\left(J(T) \frac{\partial T}{\partial y}\right)-\frac{1}{\rho c_{p}} \frac{\partial q_{r}}{\partial y} \\
+\frac{\tau}{\rho c_{p}}\left(D_{B} \frac{\partial T}{\partial y} \frac{\partial C}{\partial y}+\frac{D_{T}}{T_{\infty}}\left(\frac{\partial T}{\partial y}\right)^{2}\right)+\frac{\mu}{\rho c_{p}}\left(\frac{\partial u}{\partial y}\right)^{2}, \\
\frac{\partial C}{\partial t}+u \frac{\partial C}{\partial x}+v \frac{\partial C}{\partial y}=D_{B} \frac{\partial^{2} C}{\partial y^{2}}+\frac{D_{T}}{T_{\infty}} \frac{\partial^{2} T}{\partial y^{2}}-K_{n}\left(C-C_{\infty}\right) .
\end{gathered}
$$

The dimensional boundary conditions are:

1. Blasius problem:

$$
\begin{array}{rc}
u=0, v=0 & \text { at } y=0 \\
u=U & \text { as } y \rightarrow \infty
\end{array}
$$

2. Sakiadis problem:

$$
\begin{aligned}
u=U, v=0 & \text { at } y=0, \\
u=0 & \text { as } y \rightarrow \infty
\end{aligned}
$$

$$
T=T_{w}, \quad C=C_{w} \text { at } y=0, \quad T=T_{\infty}, C=C_{\infty}, \text { as } y \rightarrow \infty .
$$

The thermal conductivity is assumed to linearly vary with temperature, (Narsu and Kumar, 2018) as:

$$
J(T)=J_{\infty}\left(1+\epsilon\left(\frac{T-T_{\infty}}{T_{w}-T_{\infty}}\right)\right),
$$

$\epsilon$ is a small parameter known as the variable conductivity parameter, and $J_{\infty}$ is the thermal conductivity of the fluid far away from the sheet. The radiative heat flux following Hossain et al. (1999) and Raptis (1998), can be expressed(Roseland approximation) as

$$
q_{r}=-\frac{4 \sigma^{*}}{3 \kappa^{*}} \frac{\partial T^{4}}{\partial y} .
$$

Here, $\sigma^{*}$ and $\kappa^{*}$ are respectively, the Stephan-Boltzmann constant and the mean absorption coefficient. We assume that the temperature different within flow is such that the term $T^{4}$ can be expressed as a linear function of temperature. Hence, expanding $T^{4}$ in Taylor series about $T_{\infty}$ and neglecting higher-order terms, we obtain

$$
T^{4} \approx 4 T_{\infty}^{3} T-T_{\infty}^{4}
$$

The stretching velocity is assumed as $U_{w}(x, t)=a x /(1-c t)$, where $a$ and $c$ are constants (with $a \geq 0$ and $c \geq 0$, where $c t \leq 1$ ) and both have dimension (time $)^{-1}$ and $a$ as the initial stretching rate $a /(1-c t)$ and it is increasing with time. We also assume the surface temperature, $T_{w}(x, t)$ and concentration, $C_{w}(x, t)$ of the stretching to vary with the 
distance $x$ and an inverse square law for its decrease with time in the following form:

$$
T_{w}(x, t)=T_{\infty}+\frac{b x}{(1-c t)^{2}}, \quad C_{w}(x, t)=C_{\infty}+\frac{b x}{(1-c t)^{2}},
$$

We choose these particular forms of $U_{w}(x, t), T_{w}(x, t)$ and $C_{w}(x, t)$ to obtain similarity transformation. We introduce the stream function $\psi$ which is defined in the usual way, a similarity variable $\eta$ and the following similarity transformations.

$$
\begin{array}{r}
\eta=\left(\frac{a}{\nu(1-c t)}\right)^{\frac{1}{2}} y, \quad \psi=\left(\frac{\nu a}{(1-c t)}\right)^{\frac{1}{2}} x f(\eta) \\
\theta(\eta)=\frac{T-T_{\infty}}{T_{w}-T_{\infty}}, \phi(\eta)=\frac{C-C_{\infty}}{C_{w}-C_{\infty}}
\end{array}
$$

where, $\psi(x, y, t)$ is a stream function defined as $u=\frac{\partial \psi}{\partial y}$ and $v=-\frac{\partial \psi}{\partial x}$. The engineering important characteristics of the flow are the skin friction coefficient, the local Nusselt and Sherwood numbers, defined respectively, as:

$C_{f}=\frac{\tau_{w}}{\rho U_{w}^{2} / 2}, \quad N u_{x}=\frac{x q_{w}}{k_{\infty}}\left(T_{w}-T_{\infty}\right), \quad S h_{x}=\frac{x j_{x}}{k_{\infty}\left(C_{w}-C_{\infty}\right)}$,

where $\tau_{w}=\mu\left(\frac{\partial u}{\partial y}\right)_{y=0}, q_{w}=-k_{\infty}\left(\frac{\partial T}{\partial y}+q_{r}\right), j_{3}=-k_{\infty}\left(\frac{\partial C}{\partial y}\right)_{y=0}$. Upon substituting the similarity variables into the governing equations we obtain:

$$
\begin{aligned}
& \frac{d^{3} f}{d \eta^{3}}+f \frac{d^{2} f}{d \eta^{2}}-\left(\frac{d f}{d \eta}\right)^{2}-A\left(\frac{d f}{d \eta}+\frac{\eta}{2} \frac{d^{2} f}{d \eta^{2}}\right) \pm \lambda_{T} \theta \pm \lambda_{C} \phi \\
& -(M+K) \frac{d f}{d \eta}=0, \\
& \frac{1}{P r}\left(\frac{d^{2} \theta}{d \eta^{2}}+\varepsilon \theta \frac{d^{2} \theta}{d \eta^{2}}+\varepsilon\left(\frac{d \theta}{d \eta}\right)^{2}+N_{r} \frac{d^{2} \theta}{d \eta^{2}}\right)-A\left(2 \theta+\frac{\eta}{2} \frac{d \theta}{d \eta}\right) \\
& -\left|\begin{array}{cc}
\frac{d f}{d \eta} & \frac{d \theta}{d \eta} \\
f & \theta
\end{array}\right|+N_{b} \frac{d \theta}{d \eta} \frac{d \phi}{d \eta}+N_{t}\left(\frac{d \theta}{d \eta}\right)^{2}+E_{c}\left(\frac{d^{2} f}{d \eta^{2}}\right)^{2}=0, \\
& \frac{d^{2} \phi}{d \eta^{2}}-\operatorname{LeA}\left(2 \phi+\frac{\eta}{2} \frac{d \phi}{d \eta}\right)+L e\left|\begin{array}{cc}
\frac{d \phi}{d \eta} & \frac{d f}{d \eta} \\
\phi & f
\end{array}\right|+\frac{N_{t}}{N_{b}} \frac{d^{2} \theta}{d \eta^{2}} \\
& -\operatorname{LeKr} \phi=0 .
\end{aligned}
$$

Where the following definitions:

$$
\begin{aligned}
& A=\frac{c}{a}, N_{t}=\frac{\tau D_{T}\left(T_{w}-T_{\infty}\right)}{\nu T_{\infty}}, N_{b}=\frac{\tau D_{B}\left(C_{w}-C_{\infty}\right)}{\nu} \\
& \lambda_{T}=\frac{g \beta_{T} x\left(T_{w}-T_{\infty}\right)}{U_{w}^{2}}, \lambda_{C}=\frac{g \beta_{C} x\left(C_{w}-C_{\infty}\right)}{U_{w}^{2}}, N_{r}=\frac{16 \sigma^{*} T_{\infty}^{3}}{3 \kappa^{*} J_{\infty}}, \\
& E_{c}=\frac{U_{w}^{2}}{c_{p}\left(T_{w}-T_{\infty}\right)}, M=\frac{\sigma B_{0}^{2}(1-c t)}{a \rho} \\
& K=\frac{\varphi(1-c t)}{a K_{o}}, \operatorname{Pr}=\frac{\rho \nu c_{p}}{J_{\infty}}, L e=\frac{\nu}{D_{B}} .
\end{aligned}
$$

The dimensionless boundary conditions (5) - (9) becomes:

1. Blasius problem:

$$
\begin{array}{r}
f(0)=0, f^{\prime}(0)=0, \theta(0)=1, \phi(0)=1 \quad \text { at } \eta=0 \\
f^{\prime}(\infty)=1, \theta(\infty)=0, \phi(\infty)=0 \quad \text { as } \eta \rightarrow \infty
\end{array}
$$

2. Sakiadis problem:

$$
\begin{aligned}
f(0)=0, f^{\prime}(0)=1, \theta(0)=1, \phi(0)=1 & \text { at } \eta=0, \\
f^{\prime}(\infty)=0, \theta(\infty)=0, \phi(\infty)=0 & \text { as } \eta \rightarrow \infty
\end{aligned}
$$

\section{NUMERICAL SOLUTION OF THE PROBLEM}

In this work we use the spectral quasi-linearization method (SQLM) to solve the system of non-linear ordinary differential equations (16) - (18) subject to boundary conditions (19) - (22). The SQLM introduced by Motsa et al. Motsa (2013) is a combination of a quasi-linearization method (QLM) and Chebyshev spectral collocation method. The QLM, originally proposed by Bellman and Kalaba (1965), is generally a Newton-Raphson based method for solving functional equations. The iterative scheme obtained from the QLM is then solved using the Chebyshev pseudospectral method. For further reading on the derivation of the quasi-linearization scheme we refer the interested reader to Motsa et al. (2014). Applying the SQLM on the system of non-linear ordinary differential equations (16) (18) gives an iterative sequence of linear equations:

$$
\begin{aligned}
& a_{0, s} \frac{d^{3} f_{s+1}}{d \eta^{3}}+a_{1, s} \frac{d^{2} f_{s+1}}{d \eta^{2}}+a_{2, s} \frac{d f_{s+1}}{d \eta}+a_{3, s} f_{s+1} \pm a_{4, s} \theta_{s+1} \\
& \pm a_{5, s} \phi_{s+1}=R_{1, s}, \\
& b_{0, s} \frac{d^{2} \theta_{s+1}}{d \eta^{2}}+b_{1, s} \frac{d \theta_{s+1}}{d \eta}+b_{2, s} \theta_{s+1}+b_{3, s} \frac{d^{2} f_{s+1}}{d \eta^{2}}+b_{4, s} \frac{d f_{s+1}}{d \eta} \\
& +b_{5, s} f_{s+1}+b_{6, s} \frac{d \phi_{s+1}}{d \eta}=R_{2, s} \\
& c_{0, s} \frac{d^{2} \phi_{s+1}}{d \eta^{2}}+c_{1, s} \frac{d \phi_{s+1}}{d \eta}+c_{2, s} \phi_{s+1}+c_{3, s} \frac{d f_{s+1}}{d \eta}+c_{4, s} f_{s+1} \\
& +c_{5, s} \frac{d^{2} \theta_{s+1}}{d \eta^{2}}=R_{3, s},
\end{aligned}
$$

subject to the same boundary conditions (19) - (21) except that they are now evaluated at the current iteration level denoted by the subscript $s+1$. The variable coefficients have the following definitions:

$$
\begin{aligned}
& a_{0, s}=1, \quad a_{1, s}=f_{s}-\frac{A \eta}{2}, a_{2, s}=-2 \frac{d f_{s}}{d \eta}-A-M-K, \\
& a_{3, s}=\frac{d^{2} f_{s}}{d \eta^{2}}, a_{4, s}=\lambda_{T}, \\
& a_{5, s}=\lambda_{C}, \quad b_{0, s}=\frac{1}{P r}+\frac{\epsilon \theta_{s}}{P r}+\frac{N r}{P r}, \\
& b_{1, s}=\frac{2 \epsilon}{\operatorname{Pr}} \frac{d \theta_{s}}{d \eta}-\frac{A \eta}{2}+f_{s}+N_{b} \frac{d \phi_{s}}{d \eta}+2 N_{t} \frac{d \theta_{s}}{d \eta}, \\
& b_{2, s}=\frac{\epsilon}{\operatorname{Pr}} \frac{d^{2} \theta_{s}}{d \eta^{2}}-2 A-\frac{d f_{s}}{d \eta}, \\
& b_{3, s}=2 E_{c} \frac{d^{2} f_{s}}{d \eta^{2}}, \quad b_{4, s}=-\theta_{s}, \quad b_{5, s}=\frac{d \theta_{s}}{d \eta}, b_{6, s}=N_{b} \frac{d \theta_{s}}{d \eta} \\
& c_{0, s}=1, \quad c_{1, s}=L e f_{s}-L e \frac{A \eta}{2}, \quad c_{2, s}=-2 L e A-L e \frac{d f_{s}}{d \eta}-L e K_{r}, \\
& c_{3, s}=-L e \phi_{s}, \quad \\
& c_{4, s}=L e \frac{d \phi_{s}}{d \eta}, \quad c_{5, s}=L e \frac{N_{t}}{N_{b}}, R_{1, s}=f_{s} \frac{d^{2} f_{s}}{d \eta^{2}}-\left(\frac{d f}{d \eta}\right)^{2}, \\
& R_{2, s}=\frac{\epsilon \theta_{s}}{P r} \frac{d^{2} \theta_{s}}{d \eta^{2}}+\frac{\epsilon}{\operatorname{Pr}}\left(\frac{d \theta_{s}}{d \eta}\right)^{2}- \\
& \theta_{s} \frac{d f_{s}}{d \eta}+f_{s} \frac{d \theta_{s}}{d \eta}+N_{b} \frac{d \theta_{s}}{d \eta} \frac{d \phi_{s}}{d \eta}+N_{t}\left(\frac{d \theta_{s}}{d \eta}\right)^{2}+E_{c}\left(\frac{d^{2} f_{s}}{d \eta^{2}}\right)^{2}, \\
& R_{3, s}=L e f_{s} \frac{d \phi_{s}}{d \eta}-L e \phi_{s} \frac{d f_{s}}{d \eta} .
\end{aligned}
$$

To apply the spectral collocation method, it is convenient to migrate from the physical domain $[0, \infty)$ to the computational domain $[-1,1]$ using a linear transformation $x(\eta)=\frac{2}{L_{\infty}} \eta-1 . L_{\infty}$ is a sufficiently large number used to replace the semi-infinite interval $[0, \infty)$ with the closed 
interval $\left[0, L_{\infty}\right]$.

$$
\left[\begin{array}{lll}
\mathbf{A}_{11} & \mathbf{A}_{12} & \mathbf{A}_{13} \\
\mathbf{A}_{21} & \mathbf{A}_{22} & \mathbf{A}_{23} \\
\mathbf{A}_{31} & \mathbf{A}_{\mathbf{3 2}} & \mathbf{A}_{\mathbf{3 3}}
\end{array}\right]\left[\begin{array}{l}
\mathbf{F}_{s+1} \\
\boldsymbol{\Theta}_{s+1} \\
\boldsymbol{\Phi}_{s+1}
\end{array}\right]=\left[\begin{array}{l}
\mathbf{R}_{1, s} \\
\mathbf{R}_{2, s} \\
\mathbf{R}_{2, s}
\end{array}\right]
$$

where

$$
\begin{aligned}
& \mathbf{A}_{11}=\mathbf{a}_{0, s} \mathbf{D}^{3}+\mathbf{a}_{1, s} \mathbf{D}^{2}+\mathbf{a}_{2, s} \mathbf{D}+\mathbf{a}_{3, s}, \mathbf{A}_{12}=\mathbf{a}_{4, s}, \quad \mathbf{A}_{13}=\mathbf{a}_{5, s}, \\
& \mathbf{A}_{21}=\mathbf{b}_{3, s} \mathbf{D}^{2}+\mathbf{b}_{4, s} \mathbf{D}+\mathbf{b}_{5, s}, \mathbf{A}_{22}=\mathbf{b}_{0, s} \mathbf{D}^{2}+\mathbf{b}_{1, s} \mathbf{D}+\mathbf{b}_{2, s}, \\
& \mathbf{A}_{23}=\mathbf{b}_{6, s} \mathbf{D}, \mathbf{A}_{31}=\mathbf{c}_{3, s} \mathbf{D}+\mathbf{c}_{4, s}, \mathbf{A}_{32}=\mathbf{c}_{5, s} \mathbf{D}^{2}, \\
& \mathbf{A}_{33}=\mathbf{c}_{0, s} \mathbf{D}^{2}+\mathbf{c}_{1, s} \mathbf{D}+\mathbf{c}_{2, s}, \\
& \mathbf{F}_{s+1}=\left[f_{s+1}\left(\eta_{0}\right), f_{s+1}\left(\eta_{1}\right), \cdots, f_{s+1}\left(\eta_{N-1}, f_{s+1}\left(\eta_{N}\right)\right]^{T},\right. \\
& \boldsymbol{\Theta}_{s+1}=\left[\theta_{s+1}\left(\eta_{0}\right), \theta_{s+1}\left(\eta_{1}\right), \cdots, \theta_{s+1}\left(\eta_{N-1}, \theta_{s+1}\left(\eta_{N}\right)\right]^{T},\right. \\
& \mathbf{\Phi}_{s+1}=\left[\phi_{s+1}\left(\eta_{0}\right), \phi_{s+1}\left(\eta_{1}\right), \cdots, \phi_{s+1}\left(\eta_{N-1}, \phi_{s+1}\left(\eta_{N}\right)\right]^{T} .\right.
\end{aligned}
$$

\section{RESULTS AND DISCUSSION}

The differential equations under study were solved using SQLM. The convergence of the SQLM solution was confirmed by the use of solution based errors. These errors are obtained by computing the differences between approximate solutions at the previous and current iteration levels $s$ and $s+1$, respectively. The error norms are defined as follows:

$$
\begin{aligned}
& \operatorname{Error}_{f}=\max _{0 \leq i \leq N}\left\|F_{s+1, i}-F_{s, i}\right\|_{\infty}, \\
& \operatorname{Error}_{\theta}=\max _{0 \leq i \leq N}\left\|\theta_{s+1, i}-\theta_{s, i}\right\|_{\infty}, \\
& \operatorname{Error}_{\phi}=\max _{0 \leq i \leq N}\left\|\phi_{s+1, i}-\phi_{s, i}\right\|_{\infty} .
\end{aligned}
$$

Figure 1 shows that the error in each of the functions $f, \theta$ and $\phi$ decreases with increasing number of iterations. The solutions converge after the 6 th, 7th and 8th iterations, respectively.

For all the numerical results in this work, the default parameter values considered are: $\lambda_{T}=3, \lambda_{C}=1, N b=0.2, \epsilon=0.2, A=0.2$, $M=0.5, L e=0.3$.

Figure 2 displays the effects of the unsteadiness $(A)$ on the velocity profiles of both the Blasius and Sakiadis flows. Though the flows behave differently, the velocity profiles are reduced when the unsteadiness parameter increases. We observe that stretching of sheet can be used as a flow stabilizer as the boundary layer thicknesses are reduced with increasing values of the unsteadiness parameter. Figures 3 and 4 show how the temperature and concentration profiles are respectively, influenced by the unsteadiness parameter. We observe that both boundary layer thickness are reduced with increasing values of the unsteadiness parameter A. In both figures we notice that the Blasius flow has thinker boundary layers flow than the Sakiadis flow. As the unsteadiness parameter comes as the sheet is stretching, increasing its values implies the increase of the surface area over which the fluid flows. This explains why the fluid properties like velocity, temperature concentration are reduced as depicted in Figures 2, 3 and 4.

Figure 5 depicts the effect of varying the values of the variable thermal conductivity parameter. The fluid velocity is greatly enhanced by increasing the thermal conductivity of the fluid in both Blasius and Sakiadis flows. As can be seen on Figures 6 and 7 an increase in the thermal conductivity of the flow system enhances the rate of heat transfer thus causing the thickening of both the thermal and solutal boundary layers. Increasing the values of the thermal conductivity implies the increase of more temperature distribution within the flow system this then physically results in the thickening of the thermal boundary layer. Physically, heat generation within the fluid, lightens the fluid particles thus increasing the fluid velocity in both cases. However, though it has little effect on the concentration, the heating of the fluid implies acceleration processes like chemical reaction which in turn reduces the fluid concentration within the flow system. The influence of the Brownian motion parameter $\mathrm{Nb}$ on the fluid velocity profiles on both types of flow is depicted on Figure

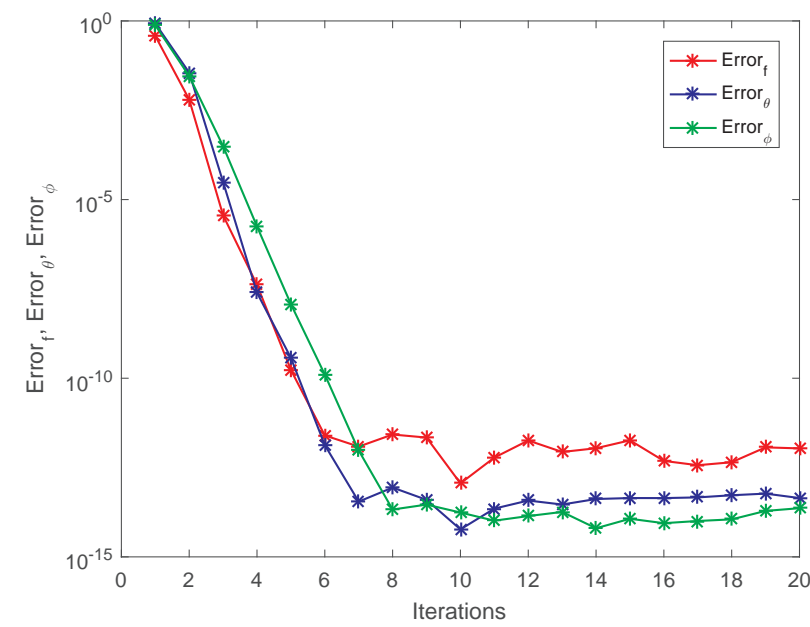

Fig. 1 Infinity error norms against iterations

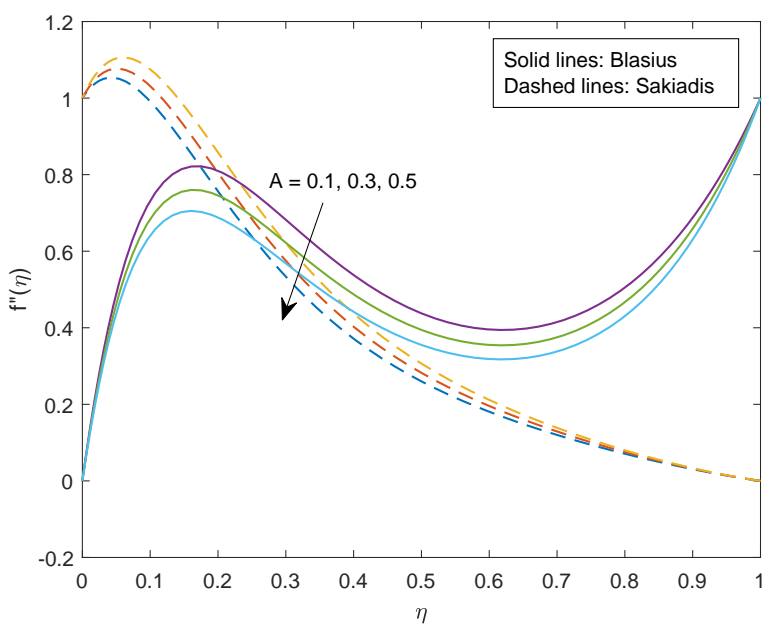

Fig. 2 The influence of $A$ on the velocity profiles

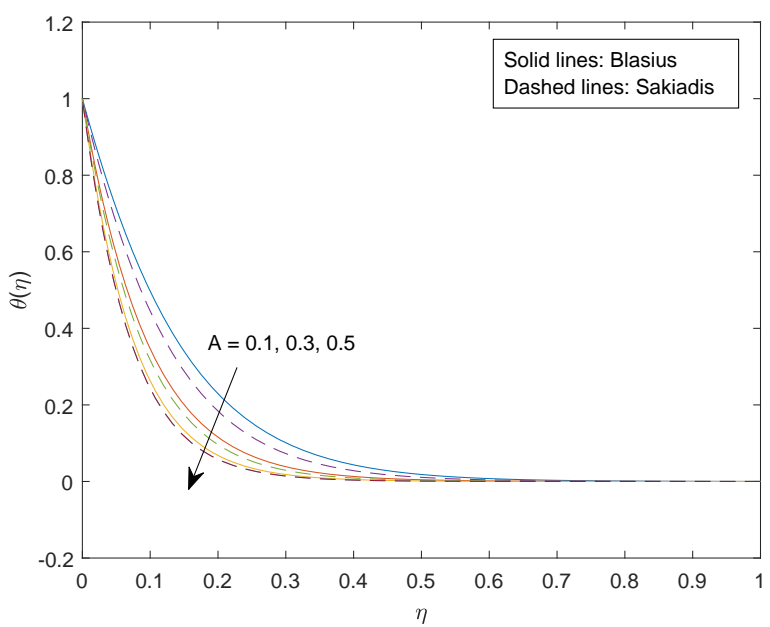

Fig. 3 The influence of $A$ on the temperature profiles

8. The velocity profiles are reduced as the Brownian motion parameter increases with increasing values of the Brownian motion parameter thus 


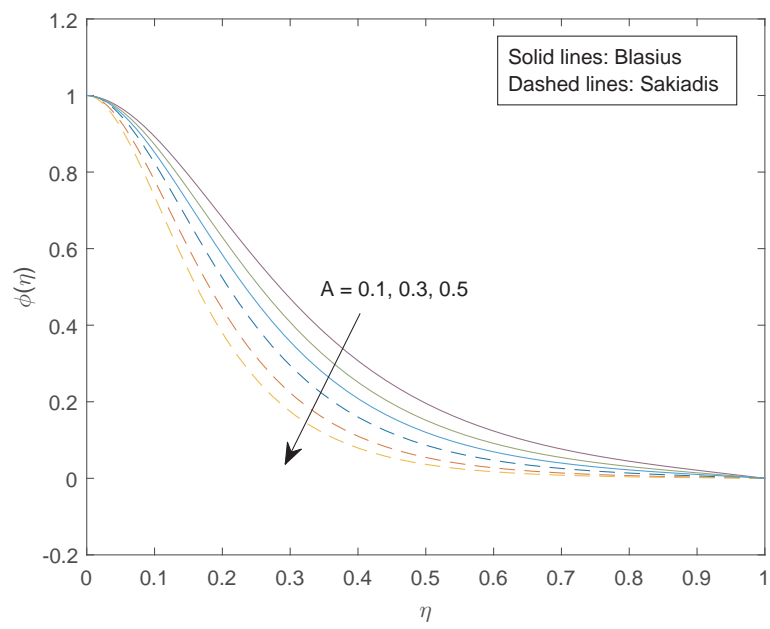

Fig. 4 The influence of $A$ on the concentration profiles

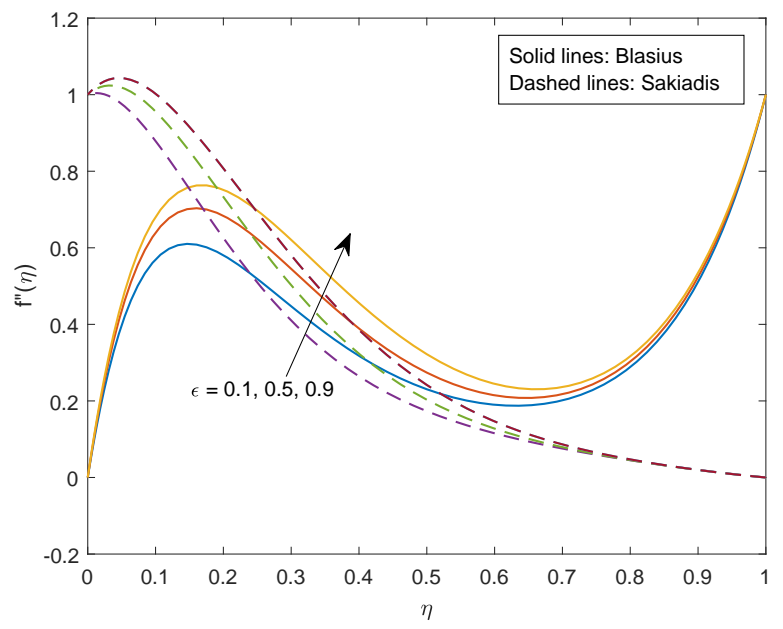

Fig. 5 The influence of $\epsilon$ on the velocity profiles

increases the fluid temperature, Figure 9 . We clearly observe in Figure 10 that increasing the values of the Brownian motion parameter causes a sharp increase of the concentration profiles near the surface wall. The influence is significantly clear on the Blasius flow. However, away from the wall the concentration profiles decrease with increasing values of the Brownian motion. Physically, Brownian motion is the random motion of particles suspended in a fluid. Therefore increasing the Brownian motion parameter results in the slowing down of the overall fluid flow. This can be likened to traffic congestion. However, the collision of particles with the fast moving molecules within the fluid results in the heat generation thus increasing the temperature distribution within the system.

Figure 11 depicts the influence of the Eckert number on the velocity profiles. We notice that in both the Blasius and Sakiadis flows, there are sharp increases of the velocity profiles as the Eckert number increases. Though the Eckert number does not directly influence the momentum equation but through the energy equation it has significant influence on the velocity profile. Physically, improving the dissipation causes the interaction among the particles less therefore this causes the velocity profiles to be depressed. We also remark that increasing the values of the Eckert number generates heat energy in the fluid due to frictional heating. This in urn enhances the temperature at any given point as can be clearly see in Figure 12.

The influence of the thermophoresis parameter on the velocity pro-

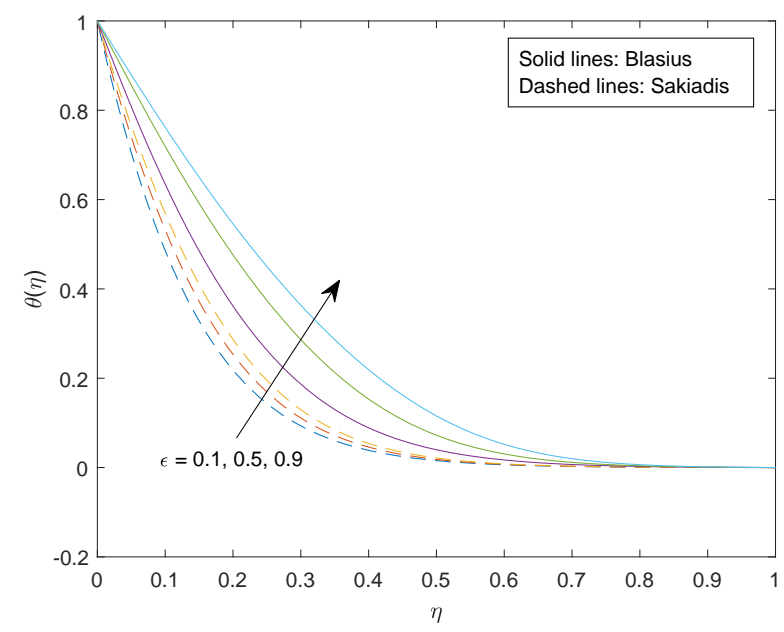

Fig. 6 The influence of $\epsilon$ on the temperature profiles

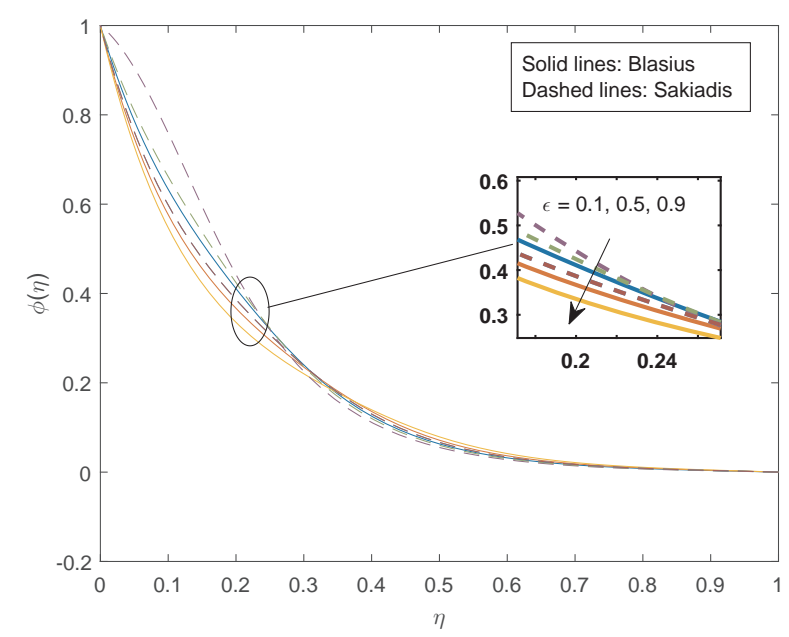

Fig. 7 The influence of $\epsilon$ on the concentration profiles

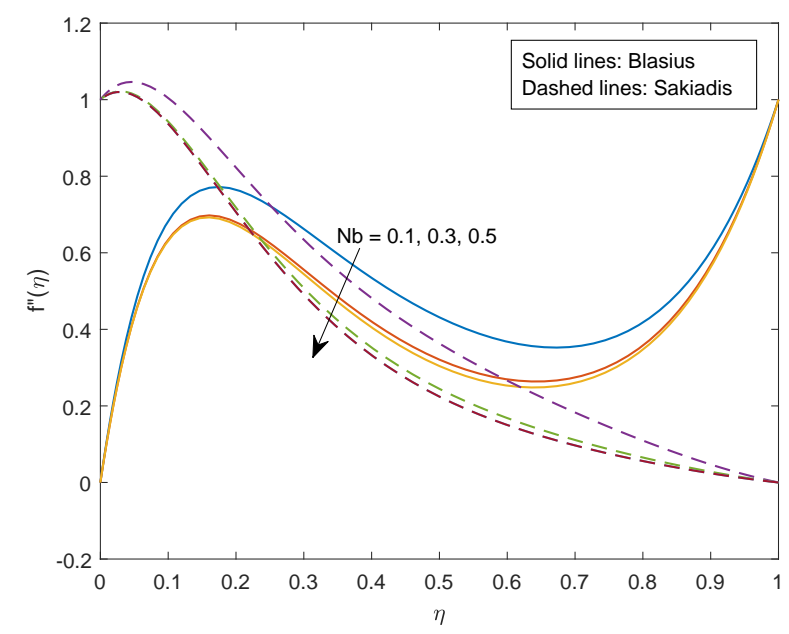

Fig. 8 The influence of $N b$ on the velocity profiles

files is depicted on Figure 13. The velocity profiles are increasing as the values of the thermophoresis parameter $\mathrm{Nt}$, increase. We also observe 


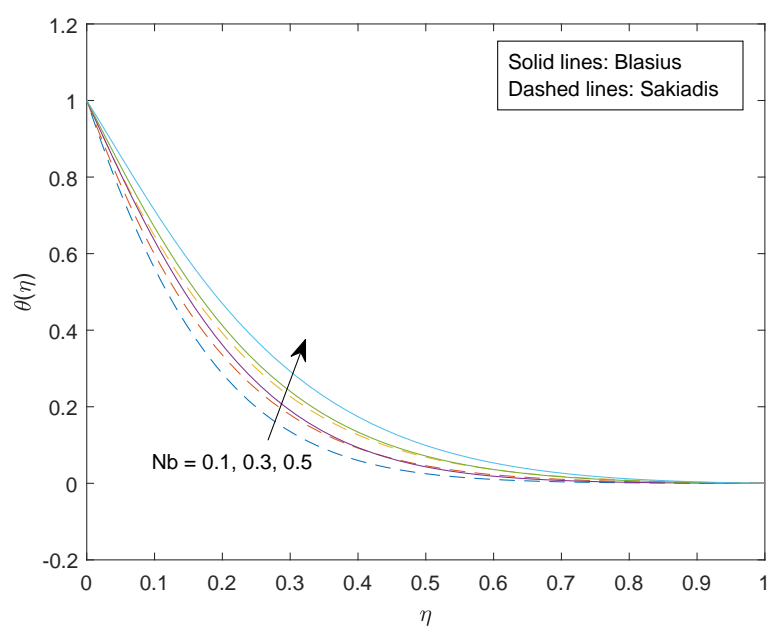

Fig. 9 The influence of $N b$ on the temperature profiles

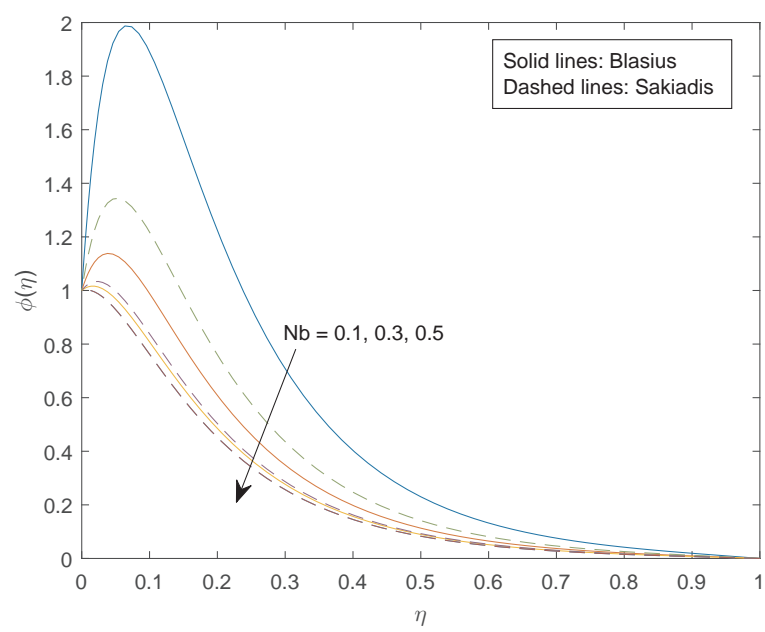

Fig. 10 The influence of $N b$ on the concentration profiles

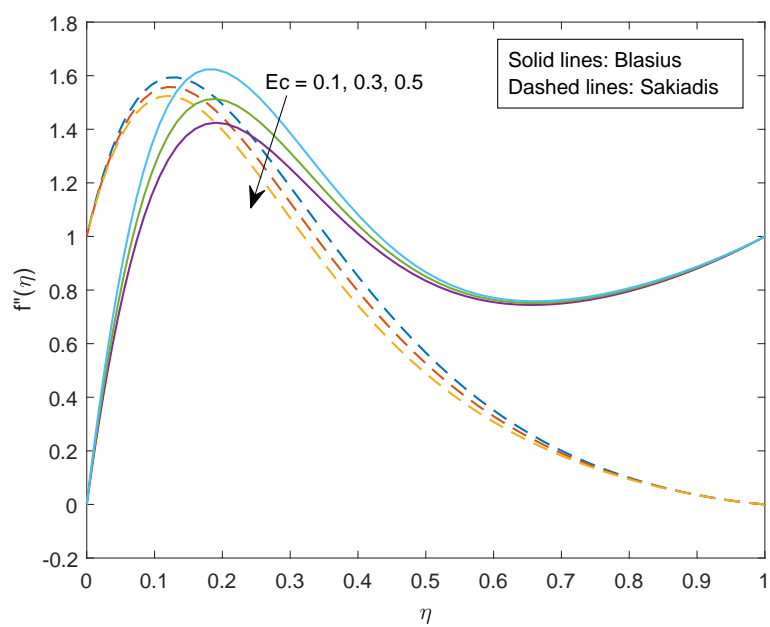

Fig. 11 The influence of $E c$ on the velocity profiles

from Figures 14 and 15, that the temperature and solutal boundary layers are significantly increased by increasing values of the thermophoresis

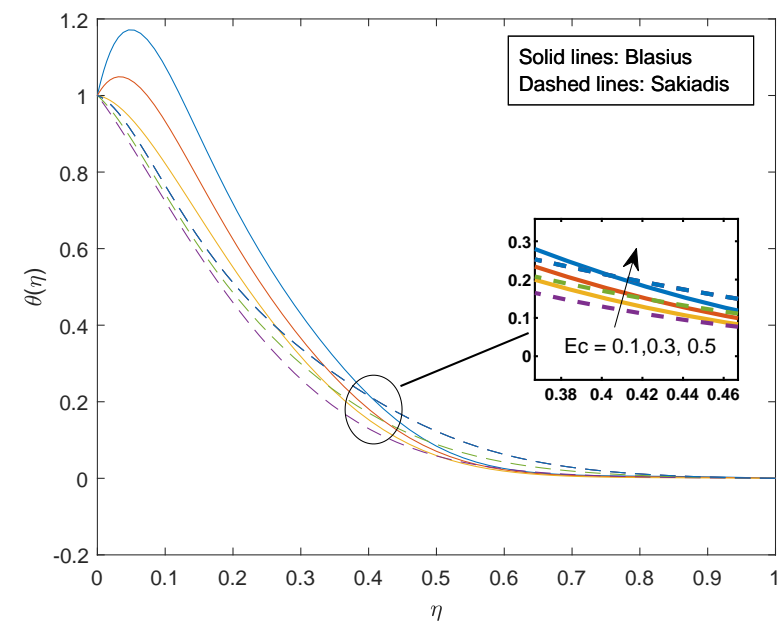

Fig. 12 The influence of $E c$ on the temperature profiles

parameter. Physically, the force induced by thermophoresis has the tendency to diffuse the particles from hotter to cooler areas thus increasing the temperature and concentration distribution within the fluid flow.

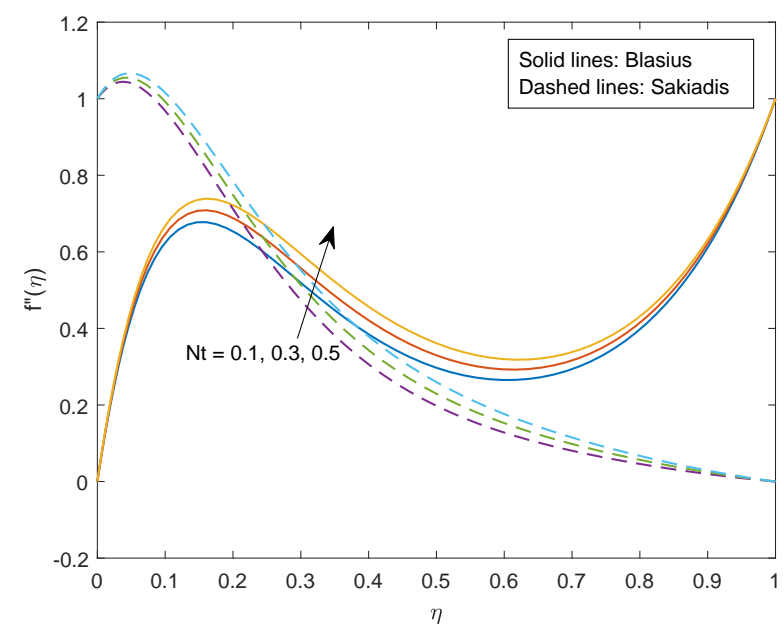

Fig. 13 The influence of $N t$ on the velocity profiles

Figures 16 and 17 display the influence of the thermal radiation parameter on the velocity and temperature profiles in both two types of flows. It is clearly observed that the thermal radiation parameter significantly enhances both the velocity and temperature profiles. This is because increasing the values of the thermal radiation parameter results in the generation of heat energy in the flow.

Figures 18 - 20 respectively, depict the influence of the porous parameter $K$ on the fluid velocity, temperature and concentration. It is clearly seen on Figure 18 that the dimensionless velocity decreases for both cases of Blasius and Sakiadis characteristics. Physically, it must be noted that as $K$ increases, the porous medium becomes tighter, therefore the resistance against the flow increases. Thus this reduction in fluid velocity makes more heat and concentration of the fluid to increase as displayed in Figures 19 and 20.

Table 1 displays the influence of the unsteadiness parameter, magnetic parameter, thermophoresis, Eckert number as well as the Lewis number on the skin friction, heat transfer and mass transfer causes the skin friction as well as the Nusselt number to be increased which the Sherwood number is reduced in the case of the Sakiadis flow. However, 


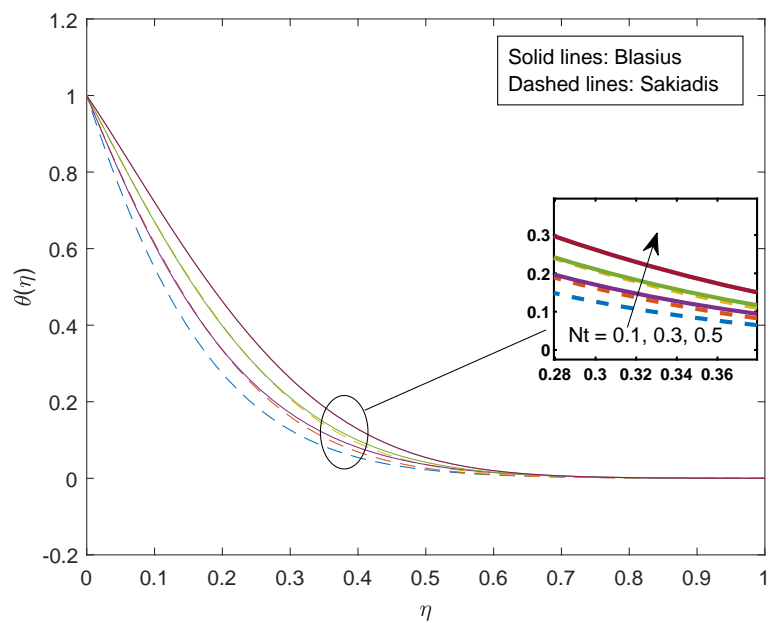

Fig. 14 The influence of $N t$ on the temperature profiles

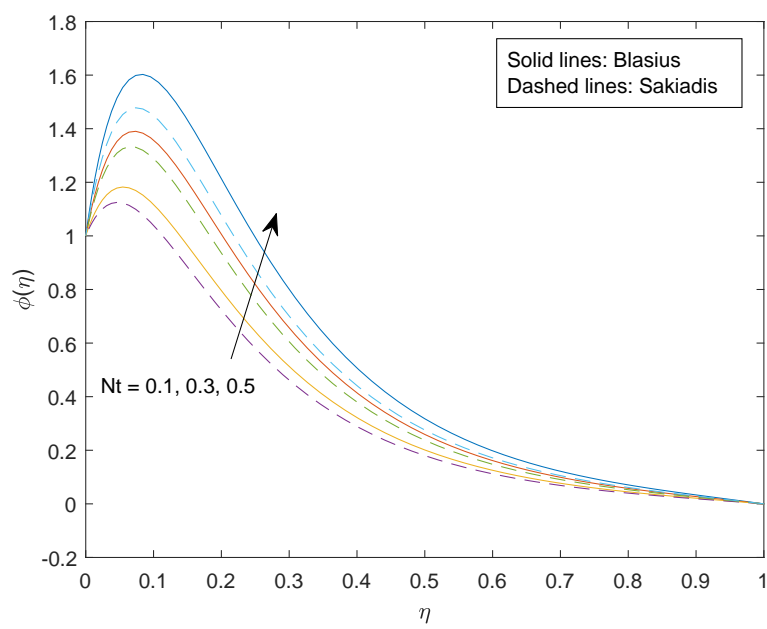

Fig. 15 The influence of $N t$ on the concentration profiles

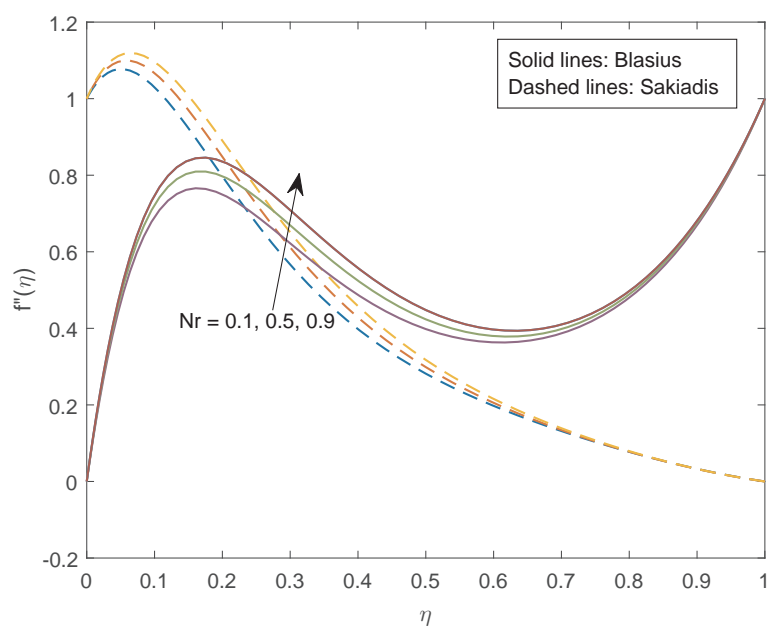

Fig. 16 The influence of $N r$ on the velocity profiles

an opposite trend happens in the case of the Blasius flow Table 2. The magnetic field, Lewis number and the Brownian motion parameters en-

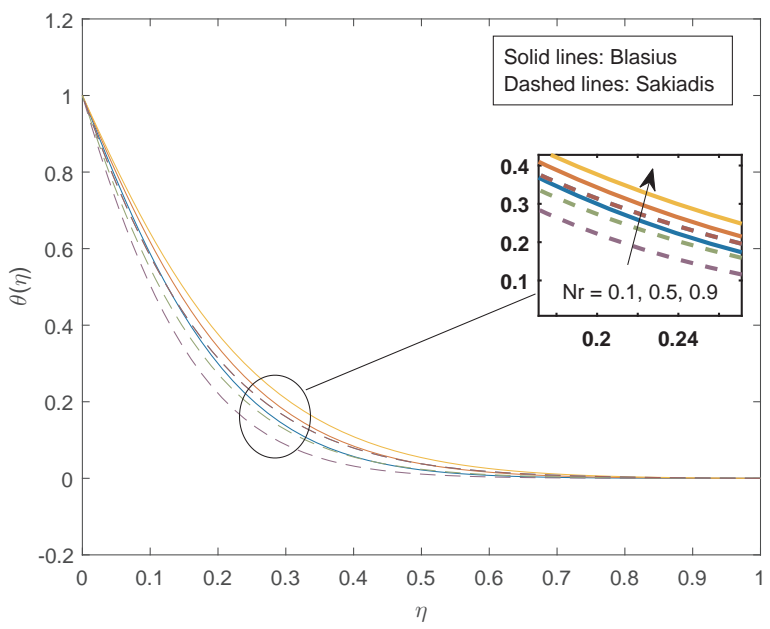

Fig. 17 The influence of $N r$ on the temperature profiles

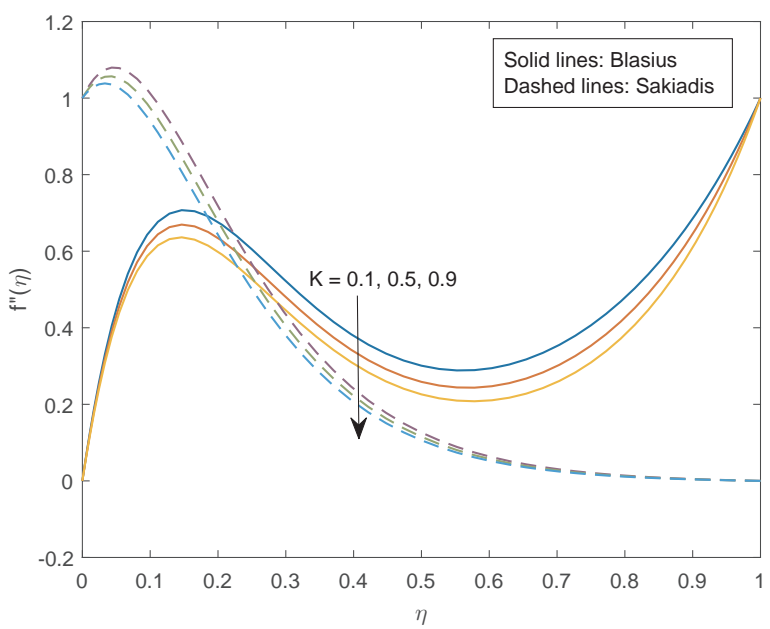

Fig. 18 The influence of $K$ on the velocity profiles

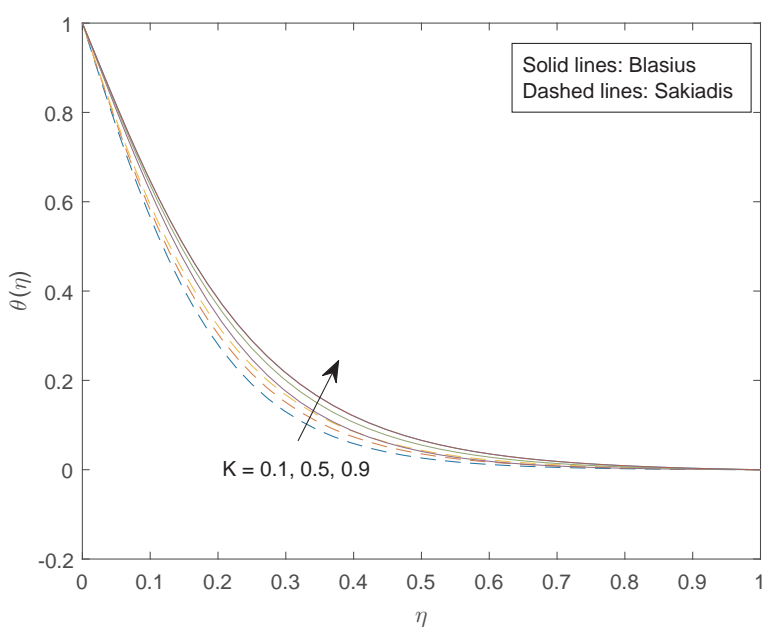

Fig. 19 The influence of $K$ on the temperature profiles

chance the skin friction coefficient while it is reduced by respectively, increases of the values of the Eckert number and thermophoresis param- 


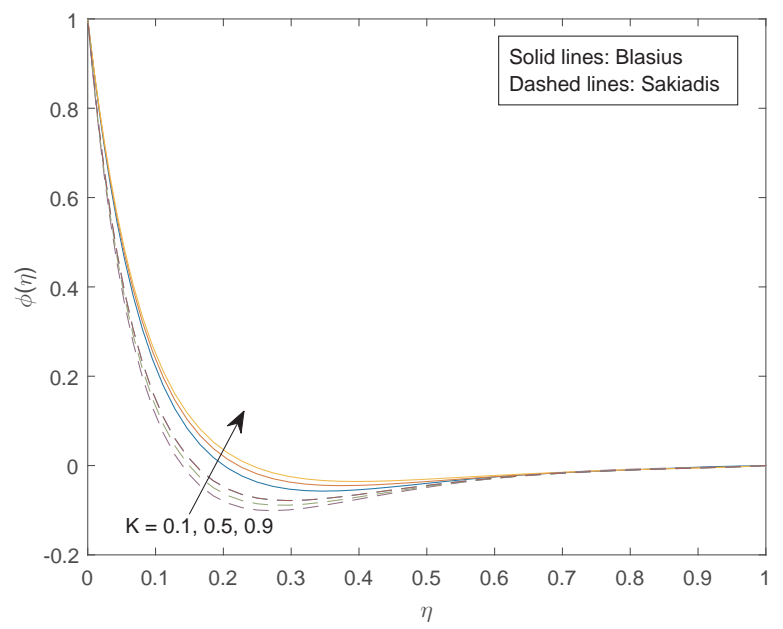

Fig. 20 The influence of $K$ on the concentration profiles

eter in the Sakiadis case. However, the opposite trend of the influences of these parameter is observed in the Blasius flow case. We clearly observe in both flows that the Sherwood number is increased by increasing the values of the magnetic parameter as well as the Lewis number.

Table 1 The values of $f^{\prime \prime}(0, \xi),-\theta^{\prime}(0, \xi)$ and $-\phi^{\prime}(0, \xi)$ for different values of the parameters $\mathrm{A}, \mathrm{M}, \epsilon, N_{b}, E_{c}$ and $\mathrm{K}$ for Sakiadis flow.

\begin{tabular}{|c|c|c|c|c|c|c|c|c|}
\hline $\mathrm{A}$ & $\mathrm{M}$ & $\mathrm{Nt}$ & $\mathrm{Nb}$ & $\overline{E_{c}}$ & Le & $-f^{\prime \prime}(0, \xi)$ & $-\theta^{\prime}(0, \xi)$ & $-\phi^{\prime}(0, \xi)$ \\
\hline 0.1 & & & & & & 0.9131 & 0.6282 & 0.2242 \\
\hline 0.3 & & & & & & 1.0029 & 0.6805 & 0.2167 \\
\hline 0.5 & & & & & & 1.0872 & 0.7293 & 0.2111 \\
\hline & 0.5 & & & & & 0.9587 & 0.6805 & 0.2167 \\
\hline & 1.0 & & & & & 1.1526 & 0.6628 & 0.2190 \\
\hline & 1.5 & & & & & 1.3270 & 0.6477 & 0.2211 \\
\hline & & 0.1 & & & & 0.9783 & 0.6883 & 0.3683 \\
\hline & & 0.3 & & & & 0.9587 & 0.6805 & 0.2167 \\
\hline & & 0.5 & & & & 0.9399 & 0.6727 & 0.0712 \\
\hline & & & 0.1 & & & 0.8375 & 0.7359 & 0.8424 \\
\hline & & & 0.3 & & & 0.9402 & 0.6995 & 0.4667 \\
\hline & & & 0.5 & & & 0.9587 & 0.6805 & 0.2167 \\
\hline & & & & 0.1 & & 0.9584 & 0.6680 & 0.2239 \\
\hline & & & & 0.3 & & 0.9576 & 0.6429 & 0.2384 \\
\hline & & & & 0.5 & & 0.9569 & 0.6179 & 0.2528 \\
\hline & & & & & 0.1 & 0.9587 & 0.6805 & 0.2167 \\
\hline & & & & & 0.3 & 0.9982 & 0.6563 & 0.5587 \\
\hline & & & & & 0.5 & 1.0241 & 0.6410 & 0.8118 \\
\hline
\end{tabular}

\section{CONCLUSION}

The current problem investigates the influence of significant parameters on fluid velocity, temperature and concentration as the skin friction, the Nusselt and Sherwood numbers. The governing partial differential equations are first reduced into a system of nonlinear ordinary differential equations. The resultant ordinary differential equations are then numerically by an efficient spectral quasi-linearization method. This method was found to be very accurate with tolerance levels of between $10^{12}$ and $10^{-14}$. Based on the current investigation, the following main observations are made:

1. The hydrodynamic boundary layer decreases with increasing val-
Table 2 The values of $f^{\prime \prime}(0, \xi),-\theta^{\prime}(0, \xi)$ and $-\phi^{\prime}(0, \xi)$ for different values of the parameters $\mathrm{A}, \mathrm{M}, \epsilon, N_{b}, E_{c}$ and $\mathrm{K}$ for Blasius flow.

\begin{tabular}{|c|c|c|c|c|c|c|c|c|}
\hline $\mathrm{A}$ & $\mathrm{M}$ & $\mathrm{Nt}$ & $\mathrm{Nb}$ & $E_{c}$ & Le & $-f^{\prime \prime}(0, \xi)$ & $-\theta^{\prime}(0, \xi)$ & $-\phi^{\prime}(0, \xi)$ \\
\hline 0.1 & & & & & & 0.5378 & 0.3974 & 0.2875 \\
\hline 0.3 & & & & & & 0.5083 & 0.5213 & 0.2613 \\
\hline 0.5 & & & & & & 0.4829 & 0.6280 & 0.2452 \\
\hline & 0.5 & & & & & 0.6381 & 0.4905 & 0.2701 \\
\hline & 1.0 & & & & & 0.5716 & 0.4742 & 0.2715 \\
\hline & 1.5 & & & & & 0.5225 & 0.4619 & 0.2729 \\
\hline & & 0.1 & & & & 0.5123 & 0.4695 & 0.3455 \\
\hline & & 0.3 & & & & 0.5225 & 0.4619 & 0.2729 \\
\hline & & 0.5 & & & & 0.5321 & 0.4544 & 0.2058 \\
\hline & & & 0.1 & & & 0.6444 & 0.5076 & 0.7300 \\
\hline & & & 0.3 & & & 0.5482 & 0.4706 & 0.6315 \\
\hline & & & 0.5 & & & 0.5321 & 0.4544 & 0.2059 \\
\hline & & & & 0.1 & & 0.5226 & 0.4588 & 0.2745 \\
\hline & & & & 0.3 & & 0.5223 & 0.4525 & 0.2778 \\
\hline & & & & 0.5 & & 0.5233 & 0.4462 & 0.2812 \\
\hline & & & & & 0.1 & 0.5225 & 0.4619 & 0.2729 \\
\hline & & & & & 0.3 & 0.4979 & 0.4463 & 0.5000 \\
\hline & & & & & 0.5 & 0.4814 & 0.4364 & 0.6675 \\
\hline
\end{tabular}

ues of the stretching parameter, the magnetic parameter, the Brownian motion parameter, and Eckert number, Lewis number and porosity parameter.

2. The fluid velocity increases as the values of variable thermal conductivity parameter, the thermal radiation parameter as well the thermophoresis parameter.

3. The fluid temperature is an increasing function of the magnetic, porosity, thermal conductivity, thermal radiation, Brownian motion parameters as well as the Eckert number.

4. The stretching parameter, the thermal conductivity, the Brownian motion as well as the Lewis number were found to reduce the fluid concentration.

5. The concentration was observed to be increasing as the values of the magnetic, thermophoresis and porosity parameters.

6. The magnetic field parameter, the Lewis number and the Brownian motion enhance the skin friction coefficient whilst it is reduced by respectively, increasing the values of the Eckert number and thermophoresis in the Sakiadis case. However, the opposite trend of the influences of these parameters is observed in the Blasius flow case.

We observed in the current study that the most dominating parameters on the fluid properties are unsteadiness parameter, the thermal conductivity parameter, the Brownian motion parameter and the porosity parameter. Lastly, we recommend that the since both the Blasius and the Sakiadis flows are similarly affected or influenced by all the parameters analysed in the current problem, there's really no need to investigate them simultaneously.

\section{ACKNOWLEDGEMENTS}

The authors wish to acknowledge financial support from the University of Venda. 


\section{NOMENCLATURE}

A unsteadiness parameter

a non negative constant $\left(s^{-1}\right)$

$b \quad$ non negative constant $\mathrm{K} / \mathrm{m}$

$c \quad$ non negative constant $\left(s^{-1}\right)$

$C \quad$ fluid concentration mass $(\mathrm{kg} / \mathrm{kmol})$

$C_{\infty} \quad$ ambient fluid concentration $(\mathrm{kg} / \mathrm{kmol})$

$c_{p} \quad$ specific heat $(\mathrm{J} / \mathrm{kg} \cdot \mathrm{K})$

$D_{B} \quad$ Brownian diffusion constant $\left(\mathrm{m}^{2} / \mathrm{s}\right)$

$D_{m} \quad$ mass diffusivity $\mathrm{m}^{2} / \mathrm{s}$

$D_{T} \quad$ thermophoretic diffusion coefficient $\left(\mathrm{m}^{2} / \mathrm{s}\right)$

Ec $\quad$ Eckert number

$g$ acceleration due to gravity $\mathrm{m} / \mathrm{s}^{2}$

$K \quad$ porous medium parameter $m^{2}$

$J(T) \quad$ variable thermal conductivity $(\mathrm{W} / \mathrm{m} \cdot \mathrm{K})$

$J_{\infty} \quad$ thermal conductivity far away from the sheet $(\mathrm{W} / \mathrm{m} \cdot \mathrm{K})$

Le Lewis number

$M \quad$ magnetic parameter

$N_{b} \quad$ Brownian motion parameter

$N_{r} \quad$ thermal radiation parameter

$N_{t} \quad$ thermophoresis parameter

$\operatorname{Pr} \quad$ Prandtl number

$q_{w} \quad$ heat flux $W / m^{2}$

$t \quad$ time (s)

$T \quad$ fluid temperature (K)

$T_{\infty} \quad$ ambient fluid temperature $(\mathrm{K})$

$u \quad$ velocity in the $x$-direction $(\mathrm{m} / \mathrm{s})$

$U_{w}(x, t) \quad$ velocity of the stretched sheet

$v \quad$ velocity component in the $y$-direction

$x \quad$ coordinate $(\mathrm{m})$

$y \quad$ coordinate $(\mathrm{m})$

Greek Symbols

$\beta \quad$ coefficient of thermal viscosity

$\varepsilon \quad$ a small parameter known as variable conductivity parameter

$\rho \quad$ fluid density $\left(\mathrm{kg} / \mathrm{m}^{3}\right)$

$\sigma^{*} \quad$ Stefan-Boltzmann constant $\left(W / m^{2}\right)$

$\kappa^{*} \quad$ mean absorption constant

$\nu \quad$ kinematic viscosity

$\lambda_{T} \quad$ thermal Grashof number

$\lambda_{C} \quad$ concentration Grashof number

$\psi(x, y, t)$ a stream function

Superscripts

$w \quad$ at the wall surface

$\infty \quad$ ambient environment

\section{REFERENCES}

Ahmad, K., and Ishak, A., 2017, "Magnetohydrodynamic(MHD) Jeffery fluid over a stretching vertical surface in a porous medium," Propulsion and Power Research, 6(4).

https://doi.org/10.1016/j.jppr.2017.11.00.

Aminikhah, H., and Kazemi, S., 2016, "Numerical Solution of the Blasius Viscous Flow Problem by Quartic B-Spline Method," International Journal of Engineering Mathematics, Volume 2016, 1-6.

https://doi.org/10.1155/2016/9014354.

Anuar, N., and Bachok, N., 2016, "Blasius and Sakiadis Problems in Nano fluids using using Buongiorno Model and Thermophysical Properties of Nanoliquids," European International Journal of Science and Technology, 5, 65-81.

Astuti, H., Sri, P., and Kaprawi, S., 2019, "Natural Convection of Nanofluids Past an Accelerated Vertical Plate with Variable Wall Temperature by Presence of the Radiation," Frontiers in Heat and Mass Transfer,
13(3).

https://doi.org//10.5098/hmt.13.3.

Bellman, R., and Kalaba, R., 1965, Quasilinearization and Nonlinear Boundary-Value Problems, Elsevier, New York, NY, USA. https://apps.dtic.mil/dtic/tr/fulltext/u2/605553.pdf,.

Blasius, H., 1908, "Grenzschichten in Flussigkeiten mit kleiner reibung," Zeitschrift fÃijr angewandte Mathematik und Physik, 55, 1-37. http://naca.central.cranfield.ac.uk/reports/1950/naca-tm-1256.pdf.

Buongiorno, J., 2006, "Convective transport in nano fluids," ASME Journal of Heat transfer, 128, 240-250.

Chamkha, A., 2004, "Unsteady MHD convective heat and mass transfer past a semi-infinite vertical permeable moving plate with heat absorption," Int J Eng Sci, 42, 217-230.

https://doi.org/10.1016/S0020-7225(03)00285-4.

Choi, S., 1995, "Enhancing thermal conductivity of fluids with nanoparticles," ASME Int Mech Eng Congr Exposition, 66, 99-105.

ANL/MSD/CP-84938; CONF-951135-29 ON: DE96004174; TRN: 96:001707.

Cortell, R., 2005, "Numerical solutions of the classical Blasius flat-plate problem," Appl Math Comput, 170, 706-710.

Crane, L., 1970, "Flow past a stretching plate," Z Angew Math Phys (ZAMP), 21, 645-647.

https://doi.org/10.1007/BF01587695.

Hady, F., Mohamed, R.E., Abd-Elsalam, M., and Ahmed, M.A., 2013, "The Blasius and Sakiadis Flow in a Nano fluid through a Porous Medium in the Presence Of Thermal Radiation under a Convective Surface Boundary Condition," International Journal of Engineering and Innovative Technology (IJEIT), 3(3).

Hossain, M., Alim, M., and Rees, D.A.S., 1999, "The effect of radiation on free convection from a porous vertical plate," Int JHeat Mass Transfer, 42, 181-191.

https:////doi.org/10/1016/S0017-9310(98)00097-0.

Howarth, L., 1938, Proc. R. Soc. London Ser.A, vol. 164.

Krishna, C.M., Reddy, G.V., B.Souayeh, Raju, C.S.K., Rahimi-Gorji, M., and Raju, S.S.K., 2019, "Thermal convection of MHD Blasius and Sakiadis flow with thermal convective conditions and variable properties," Microsystem Technologies, 1-14. https://doi.org/10.1007/s00542-019-04353-y.

Kuznetsov, A., and Nield, D., 2010, "Natural convective boundary-layer flow of a nano fluid past a vertical plate," International Journalof Thermal Sciences, 49, 243-247.

https://doi.org/10.1016/j.ijthermalsci.2009.07.015.

Lighthill, M., 1950, Proc. R. Soc. London Ser.A, vol. 202.

Liu, C., and Chang, J., 2008, "The Lie-group shooting method formultiple-solutions of Falkner-Skan equation under suctionin- jection conditions," International Journal of Non-Linear Mechanics, 43(9), 844851.

https://doi.org/10.1155/2008/749865.

Mabood, F., Pochai, N., and Shateyi, S., 2016, "Stagnation Point Flow of Nano fluid over a Moving Plate with Convective Boundary Condition and Magnetohydrodynamics," Journal of Engineering. 
Mjankwi, M., Masanja, V., Mureithi, E., and James, M.N., 2019, "Unsteady MHD Flow of Nano fluid with Variable Properties over a Stretching Sheet in the Presence of Thermal Radiation and Chemical Reaction," International Journal of Mathematics and Mathematical Sciences.

Mnyusiwalla, A., Daar, A., and Singer, P., 2003, "Mind the gap : science and ethics in nanotechnology," Nanotechnology, 14, R9-R13.

Motsa, S., 2013, "A New Spectral Local Linearization Method for Nonlinear Boundary Layer Flow Problems," Journal of Applied Mathematics, volume 2013, $1-15$.

https://doi.org/10.1155/2013/423628.

Motsa, S., Dlamini, P., and Khumalo, M., 2014, "Spectral Relaxation Method and Spectral Quasilinearization Method for Solving Unsteady Boundary Layer Flow Problems." Advances in Mathematical Physics, Volume 2014, 1-12.

https://doi.org/10.1155/2014/341964.

Narsu, S., and Kumar, B.R., 2018, "Comparative study of chemically reacting Blasius and Sakiadis unsteady MHD radiated flow with variable conductivity," IOP Conf Series: Journal of Physics: Conf Series 1000, $1-11$.

https://doi :10.1088/1742-6596/1000/1/012149.

Oyelakin, I., Mondal, S., and Sibanda, P., 2016, "Unsteady Casson nano fluid flow over a stretching sheet with thermal radiation, convective and slip boundary conditions," Alexandria Engineering Journal, 55. https://doi.org/10.1016/j.aej.2016.03.003.

Raptis, A., 1998, "Flow of a micropolar fluid past a continuously moving plate by the presence of radiation," Int J Heat Mass Transfer, 4.
Reddy, P.B.A., and B. Mallikarjuna, K.M.S.R., 2018, “Slip Effect on Heat and Mass Transfer in Casson Fluid with Cattaneo-Christove Heat Flux Model," Frontiers in Heat and Mass Transfer, 11(5).

https://doi.org/10..5098/hmt.11.5.

Sakiadis, B., 1961, "Boundary-layer behavior on continuous solid surfaces: I. Boundary-layer equations for two-dimensional and axisymmetric flow," AIChE J, 7, 6-8.

https://doi.org/10.1002/aic.690070108. .

Sekhar, K., Reddy, G., and Raju, C.S.K., 2017, "Blasius and Sakiadis flow of magnetohydrodynamic Maxwell fluid with exponentially decaying heat source or sink," International Journal of Research in Science and Engineering.

Sharma, P.R., Choudhary, S., and Makinde, O.D., 2017, "MHD Slip Flow and Heat Transfer over an Exponentially Stretching Permeable Sheet Embedded in a Porous Medium with Heat Source," Frontiers in Heat and Mass Transfer, 7(18), 126-136.

https://doi.org/DOI: 10.5098/hmt.13.15.

Tsou, F., Sparrow, E., and Glodstein, R., 1967, "Flow and heat transfer in the boundary layer on a continuous moving surface," Int J Heat Mass Transfer, 10, 219-235. https://doi.org/10.1016/0017-9310(67)90100-7.

Wang, L., 2004, "A new algorithm for solving classical Blasius equation," Applied Mathematics and Computation, 157(1), 1-9. https://doi.org/10.1016/j.amc.2003.06.011. 